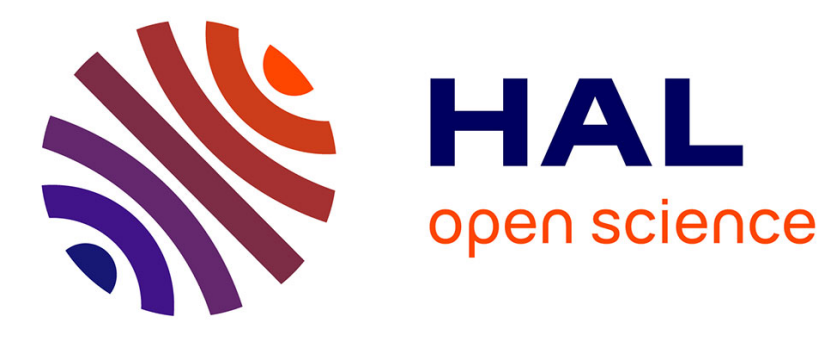

\title{
Vectorial stimulated Raman scattering resolution on the semi-line
}

\author{
Frédéric Ginovart
}

\section{To cite this version:}

Frédéric Ginovart. Vectorial stimulated Raman scattering resolution on the semi-line. Physics Letters A, 2012, 376 (34), pp.2277. 10.1016/j.physleta.2012.05.053 . hal-00710297

\section{HAL Id: hal-00710297 \\ https://hal.science/hal-00710297}

Submitted on 20 Jun 2012

HAL is a multi-disciplinary open access archive for the deposit and dissemination of scientific research documents, whether they are published or not. The documents may come from teaching and research institutions in France or abroad, or from public or private research centers.
L'archive ouverte pluridisciplinaire HAL, est destinée au dépôt et à la diffusion de documents scientifiques de niveau recherche, publiés ou non, émanant des établissements d'enseignement et de recherche français ou étrangers, des laboratoires publics ou privés. 


\title{
Vectorial stimulated Raman scattering resolution on the semi-line
}

\author{
Frédéric Ginovart ${ }^{1,2, *}$ \\ (1) UEB, Université Européenne de Bretagne, Université de Rennes I, France \\ (2) CNRS, UMR 6082 FOTON, Enssat, 6 rue de Kerampont, CS 80518, \\ 22305 Lannion cédex, France
}

\begin{abstract}
Stimulated Raman scattering between a laser pump pulse and a Stokes pulse is considered in a two-level medium with vectorial optical fields. The model on the semi-line is proved to be solvable by inverse scattering transform scheme. Among solutions, soliton generation is discussed. Then, it is shown how rotation of the Stokes wave leads to a spike of pump radiation in the time domain.
\end{abstract}

\section{Introduction}

Stimulated Raman scattering (SRS) is a well-known 3-wave interaction process and has been largely studied. It results from the interaction between a laser pump wave and a medium through a Stokes wave emission [1-2]. It is characterized by a pump depletion, e.g. an energy transfer from the pump wave to the Stokes one. Of course, this is a serious obstacle to applications where a high power pump is needed. It has also been experimentally found by Drühl and co-workers [3-4] that this energy transfer can be inversed, thus creating a spike in the pump profile. Several explanations of this phenomenon have been reported in the literature [5-10].

Among them, one is based on the inverse scattering transform (IST) [11-16]. It has already been shown that vectorial SRS is solvable on the line [13] what has allowed to explain the formation of the spike of pump radiation. Scalar SRS has also been solved on the semi-line [15] leading to a study of soliton generation. By vectorial SRS, one has to understand that optical waves are vectorial.

In this work, we show that vectorial SRS is also solvable on the semi-line. Note that this result is not obvious : a scalar nonlinear partial differential equation integrable by IST is not necessarily integrable when considering its vectorial form. This resolution allows to undertake the problem of soliton generation. Similar results to the scalar study have been obtained at the moment. Next, it is shown how a rotating Stokes polarization relatively to the pump one allows the creation of a spike of pump radiation in the time domain. As a consequence, multi-spike formation is shown to be also possible.

\section{Basics on stimulated Raman scattering}

In the SRS model with a semi-classical description, the medium is considered as a collection of harmonic oscillators interacting with an incident optical field (pump wave) through the emission of a Stokes wave. Considering slowly varying envelopes, the stimulated Raman scattering equations are:

$$
\begin{gathered}
\partial_{x} \mathbf{A}_{\mathbf{L}}=q \mathbf{A}_{\mathbf{S}} e^{2 i k x} \quad \partial_{x} \mathbf{A}_{\mathbf{S}}=-\bar{q} \mathbf{A}_{\mathbf{L}} e^{-2 i k x} \\
\partial_{t} q=-\int_{\mathbb{R}} \mathbf{A}_{\mathbf{L}} \overline{\mathbf{A}_{\mathbf{S}}} e^{-2 i k x} g(k) d k
\end{gathered}
$$

where $\mathbf{A}_{\mathbf{L}}(k, x, t)$ and $\mathbf{A}_{\mathbf{S}}(k, x, t)$ are respectively the envelopes of the laser and stokes waves while

\footnotetext{
* Corresponding author : Tel 33 (0)2 96469065 - Fax : 33 (0)2 96370199 - Email : Frederic.Ginovart@enssat.fr
} 
$\mathrm{q}(\mathrm{x}, \mathrm{t})$ is the vibrational medium wave. The variable $\mathrm{k}$ is introduced to take into account the detuning from the exact resonance, $\mathrm{x}$ and $\mathrm{t}$ are respectively the space and time variables. This system is completed with the initial condition

$$
q(x, t=0)=0
$$

expressing that the medium is initially at rest. The following boundary conditions on the laser and stokes envelopes hold

$$
\begin{aligned}
& \mathbf{A}_{\mathbf{L}}(k, x=0, t)=\left(\begin{array}{l}
I_{L}(k, t) \\
J_{L}(k, t)
\end{array}\right) \\
& \mathbf{A}_{\mathbf{s}}(k, x=0, t)=\left(\begin{array}{l}
I_{s}(k, t) \\
J_{s}(k, t)
\end{array}\right)
\end{aligned}
$$

So, in this work, we have to solve an initial boundary value problem (IVP) on the semi-line, that is $x \in[0,+\infty[$.

\section{Resolution of SRS}

Here, we are going to use the inverse scattering transform (IST) [11-12] to solve the IVP (1-3). In effect, noting that envelopes waves equations in (1) form the well-known Zakharov-Shabat problem, it seems reasonable to expect solving this problem with IST.

\section{III.1 Direct problem}

Consider the Zakharov-Shabat spectral problem:

$$
\mu_{x}+i k\left[\sigma_{3}, \mu\right]=Q \mu, \quad x \geq 0, t \geq 0, \quad Q=\left(\begin{array}{cc}
0 & q(x, t=0) \\
-\bar{q}(x, t=0) & 0
\end{array}\right)
$$

where

$$
\sigma_{3}=\left(\begin{array}{cc}
1 & 0 \\
0 & -1
\end{array}\right)
$$

and consider two fundamental solutions $\mu^{+}$and $\mu^{-}$respectively defined, in the upper and lower halfcomplex k-space. These solutions check

$$
\left(\overline{\mu_{1}^{+}(\bar{k})} \overline{\mu_{2}^{+}(\bar{k})}\right)=\left(\begin{array}{ll}
\overline{\mu_{11}^{+}(\bar{k})} & \overline{\mu_{12}^{+}(\bar{k})} \\
\overline{\mu_{21}^{+}(\bar{k})} & \overline{\mu_{22}^{+}(\bar{k})}
\end{array}\right)=\left(\begin{array}{cc}
\mu_{22}^{-}(k) & -\mu_{21}^{-}(k) \\
-\mu_{12}^{-}(k) & \mu_{11}^{-}(k)
\end{array}\right)
$$

and verify the Riemann-Hilbert relations :

$$
\mu_{1}^{+}-\mu_{1}^{-}=\rho^{-} \mu_{2}^{-} e^{2 i k x}, \quad \mu_{2}^{+}-\mu_{2}^{-}=\rho^{+} \mu_{1}^{+} e^{-2 i k x}
$$

with

$$
\rho^{+}(k)=-\int_{0}^{\infty} q(u) \mu_{22}^{+}(k, u) e^{2 i k u} d u, \quad \rho^{-}(k)=\overline{\rho^{+}(\bar{k})}
$$

The boundary conditions are given by : 


$$
\begin{gathered}
\mu^{+}(k, x=0)=\left(\begin{array}{cc}
1 & \rho^{+} \\
0 & 1
\end{array}\right) \\
\mu^{+} \underset{x \rightarrow \infty}{\longrightarrow}\left(\begin{array}{cc}
1 / \tau^{+} & 0 \\
\frac{\rho^{-}}{\tau^{-}} e^{2 i k x} & \tau^{+}
\end{array}\right)
\end{gathered}
$$$$
\mu^{-}(k, x=0)=\left(\begin{array}{cc}
1 & 0 \\
-\rho^{-} & 1
\end{array}\right)
$$$$
\mu^{-} \underset{x \rightarrow \infty}{\longrightarrow}\left(\begin{array}{cc}
\tau^{-} & -\frac{\rho^{+}}{\tau^{+}} e^{-2 i k x} \\
0 & 1 / \tau^{-}
\end{array}\right)
$$

with

$$
\tau^{+}(k)=1-\int_{0}^{\infty} \bar{q}(u) \mu_{12}^{+}(k, u) d u, \quad \tau^{-}(k)=\overline{\tau^{+}(\bar{k})}
$$

Let notice that $\rho^{+}(k)$ and $\tau^{+}(k)$ are respectively the reflection and transmission coefficient, leading to the continuous spectral data

$$
R(k)=\left\{\rho^{ \pm}, \tau^{ \pm}\right\}
$$

\section{III.2 Evolution of the spectral data}

The auxiliary spectral problem is given by

$$
R_{t}=[R, \Omega]+M
$$

where $\Omega$ and $M$ are to be found. The Lax pair is usually defined as :

$$
\left\{\begin{array}{l}
U=\left(\mu_{x}-\mu \Lambda\right) \mu^{-1}=-i k \sigma_{3}+Q \\
V=\left(\mu_{t}-\mu \Omega\right) \mu^{-1}
\end{array}\right.
$$

and the compatibility condition $\mu_{x t}=\mu_{t x}$ leads to

$$
\left\{\begin{array}{l}
\Lambda_{t}-\Omega_{x}+[\Omega, \Lambda]=0 \\
U_{t}-V_{x}+[U, V]=0
\end{array}\right.
$$

Solving equation (13a), it comes

$$
\Omega(k, x, t)=e^{-i k \sigma_{3} x} \Omega(k, t) e^{i k \sigma_{3} x}
$$

Then eq (13b) gives the time evolution of the potential:

$$
Q_{t}(x, t)=-\frac{1}{2 \pi} \iint\left[\sigma_{3}, \mu\left(M-\frac{\partial \Omega}{\partial \bar{\lambda}}\right) \mu^{-1}\right] d \lambda \wedge d \bar{\lambda}
$$

with $d \lambda \wedge d \bar{\lambda}=-2 i d \lambda_{R} d \lambda_{I}$. Thus taking into account eq (1)

$$
\partial_{t} q=-\int_{\mathbb{R}} \mathbf{A}_{\mathbf{L}} \overline{\mathbf{A}_{\mathbf{S}}} e^{-2 i k x} g(k) d k
$$

we get four equations. Next, considering the limits of $\mu_{t}$ in eq (12b) when $\mathrm{x}$ tends to 0 and to infinity, eight equations are obtained. The resulting system of 12 equations furnish the time evolution of the scattering data. For instance the time evolution of the reflection coefficient is given by the differential equation:

$$
\rho_{t}^{+}(k, t)=-\left(\rho^{+}\right)^{2} \frac{1}{\pi} \int_{\mathbb{R}} \frac{\bar{m}(\lambda, t)}{\lambda-(k+i 0)} d \lambda-2 \rho^{+} \frac{1}{\pi} \int_{\mathbb{R}} \frac{\Phi(\lambda, t)}{\lambda-(k+i 0)} d \lambda-\frac{1}{\pi} \int_{\mathbb{R}} \frac{m(\lambda, t)}{\lambda-(k+i 0)} d \lambda
$$


with $m$ and $\Phi$ are two functions to be found.

\section{III.3 Solution of SRS equations}

We have now everything to solve our problem. In effect, using boundary conditions, it is easy to link the physical solution to the mathematical one :

$$
\left\{\begin{array}{l}
\left(\begin{array}{c}
A_{L}^{1} \\
A_{S}^{1} e^{2 i k x}
\end{array}\right)=I_{L}(k, t)\left(\begin{array}{l}
\mu_{11}^{+} \\
\mu_{21}^{+}
\end{array}\right)+I_{S}(k, t)\left(\begin{array}{l}
\mu_{12}^{-} \\
\mu_{22}^{-}
\end{array}\right) e^{2 i k x} \\
\left(\begin{array}{c}
A_{L}^{2} \\
A_{S}^{2} e^{2 i k x}
\end{array}\right)=J_{L}(k, t)\left(\begin{array}{l}
\mu_{11}^{+} \\
\mu_{21}^{+}
\end{array}\right)+J_{S}(k, t)\left(\begin{array}{l}
\mu_{12}^{-} \\
\mu_{22}^{-}
\end{array}\right) e^{2 i k x}
\end{array}\right.
$$

since they both solve the Zakharov-Shabat problem. Moreover in order to get the output fields envelopes, we make $\mathrm{x}$ tends to infinity in (17) using (8). The preceding system of 12 equations allow to get explicitly the functions $m$ and $\Phi$ :

$$
\left\{\begin{array}{l}
m=\frac{i \pi}{2} g(k)\left[I_{L} \overline{I_{S}}+J_{L} \overline{J_{S}}\right] \\
\Phi=\frac{i \pi}{4} g(k)\left[\left(\left|I_{L}\right|^{2}-\left|I_{S}\right|^{2}\right)+\left(\left|J_{L}\right|^{2}-\left|J_{S}\right|^{2}\right)\right]
\end{array}\right.
$$

Then, the output field envelopes become explicit and for instance,

$$
A_{L}^{1}=\frac{1}{\tau^{+}} I_{L}(k, t)+\frac{\rho^{+}}{\tau^{+}} I_{S}(k, t)
$$

The rest of the paper is based on this formula.

\section{Examples of solution of SRS}

\section{IV.1 Soliton generation}

Let us give an example with

$$
\left\{\begin{array}{cc}
\left|I_{L}(k, t)\right|^{2}=|A(t)|^{2} f(k), & I_{S}(k, t)=\Gamma_{S} I_{L}(k, t) \\
J_{L}(k, t)=\Gamma_{L} I_{L}(k, t), & J_{S}(k, t)=\Gamma J_{L}(k, t) \\
f(k)=\frac{\kappa}{\pi\left(k^{2}+\kappa^{2}\right)} &
\end{array}\right.
$$

and $g(k)=g_{0}$, e.g., the sharp line case is considered. $\Gamma, \Gamma_{L}, \Gamma_{S}$ are the fractions of the different intensities. The solution of the Riccati differential equation (16) reads

$$
\frac{1}{\rho^{+}-\rho_{1}}=\frac{\rho_{0}-\rho_{2}}{\left(\rho_{0}-\rho_{1}\right)\left(\rho_{1}-\rho_{2}\right)} \exp \left[\left(\rho_{1}-\rho_{2}\right) \int_{0}^{t} F(u) d u\right]-\frac{1}{\rho_{1}-\rho_{2}}
$$

with 


$$
\begin{aligned}
& \rho_{0}=\rho^{+}(t=0) \quad \rho_{1}=\frac{\alpha-\sqrt{\alpha^{2}+4}}{2} \quad \rho_{2}=\frac{\alpha+\sqrt{\alpha^{2}+4}}{2} \\
& \alpha=\frac{1-\Gamma_{S}^{2}+\Gamma_{L}^{2}-\Gamma_{L}^{2} \Gamma^{2}}{\Gamma_{S}+\Gamma_{L}^{2} \Gamma} \\
& F(t)=\frac{i}{2} g_{0}\left(\Gamma_{S}+\Gamma_{L}^{2} \Gamma\right) \frac{1}{k+i \kappa}|A(t)|^{2}
\end{aligned}
$$

Let note that $\Gamma_{S}+\Gamma_{L}^{2} \Gamma>0$. When taking $\rho_{0}=0$, it comes

$$
\rho^{+}(\mathrm{t})=\frac{-2 \sinh (\Delta / 2)}{\alpha \sinh (\Delta / 2)+\sqrt{\alpha^{2}+4} \cosh (\Delta / 2)}
$$

with:

$$
\Delta=-\sqrt{\alpha^{2}+4} \int_{0}^{\mathrm{t}} \mathrm{F}(\mathrm{u}) \mathrm{du}
$$

Looking for solitons, one has to find the poles of the function $\rho^{+}$. In the case $\Gamma=\theta \Gamma_{\mathrm{S}}$, when setting

$$
\Gamma=\mathrm{e}^{-\gamma}, \Gamma_{\mathrm{S}}=\mathrm{e}^{-\gamma_{\mathrm{S}}}, \Gamma_{\mathrm{L}}=\mathrm{e}^{-\gamma_{\mathrm{L}}},
$$

one gets

$$
\alpha=2 \sinh \left(\gamma_{\mathrm{S}}\right)+\mathrm{e}^{\gamma_{\mathrm{S}}-2 \gamma_{\mathrm{L}}} \frac{1-\theta}{1+\theta \mathrm{e}^{-2 \gamma_{\mathrm{L}}}}
$$

The subcase $\theta=1$ is a particular case where the poles of the function $\rho^{+}$are given by

$$
\mathrm{k}_{\mathrm{m}}=-\mathrm{i} \kappa+\frac{\mathrm{T}(\mathrm{t})}{\left(\mathrm{m}+\frac{1}{2}\right) \pi-\mathrm{i} \gamma_{\mathrm{s}}}, \quad \mathrm{m} \in \mathbb{Z}
$$

with

$$
\mathrm{T}(\mathrm{t})=\frac{1}{4} \mathrm{~g}_{0}\left(1+\mathrm{e}^{-2 \gamma_{\mathrm{s}}}\right)\left(1+\mathrm{e}^{-2 \gamma_{\mathrm{L}}}\right) \int_{0}^{\mathrm{t}}|\mathrm{A}(\mathrm{u})|^{2} \mathrm{du}
$$

This result is very similar to the scalar SRS equations case [15] where it has been pointed that as $t$ evolves, the poles move from the point $-i \kappa$ and may cross the real axis for large enough $\mathrm{T}(\mathrm{t})$, thus generating solitons by pairs since $\mathrm{k}_{\mathrm{m}}=-\overline{\mathrm{k}}_{-\mathrm{m}-1}$.

\section{IV.2 Raman spike and multi-spike}

An interesting phenomenon occurs when considering

$$
\begin{aligned}
& \mathbf{A}_{\mathbf{L}}(k, x=0, t)=\left(\begin{array}{l}
1 \\
0
\end{array}\right) I_{L}(k, t) \\
& \mathbf{A}_{\mathbf{s}}(k, x=0, t)=\left(\begin{array}{c}
\cos \theta(t) \\
\sin \theta(t)
\end{array}\right) I_{s}(k, t)
\end{aligned}
$$

This corresponds to an injection of a linear polarized pump wave and a rotating polarized Stokes wave. Let's take 


$$
I_{S}(k, t)=e^{-\gamma} I_{L}(k, t) \text { and } \quad\left|I_{L}(k, t)\right|^{2}=|A(t)|^{2} f_{0}
$$

When taking $\rho_{0}=0$, the solution of the reflection coefficient differential equation, eq. 16 , reads

$$
\rho^{+}(\mathrm{t})=\frac{-\sinh (\Delta / 2) \cos \theta}{\sinh (\Delta / 2) \sinh (\gamma)+\sqrt{\sinh ^{2}(\gamma)+\cos ^{2} \theta} \cosh (\Delta / 2)}
$$

with:

$$
\Delta=-\frac{\pi}{2} \mathrm{~h}_{0} \mathrm{e}^{-\gamma} \int_{0}^{\mathrm{t}} \frac{|\mathrm{A}(\mathrm{u})|^{2} \cos ^{2} \theta(\mathrm{u})}{\sqrt{\sinh ^{2} \gamma+\cos ^{2} \theta(\mathrm{u})}} \mathrm{du}
$$

Let's take the input pump envelope as :

$$
I_{L}(t)=\exp \left(-\frac{\left(t-t_{1}\right)^{2}}{\tau^{2}}\right)
$$

with $\mathrm{t}_{1}=50$ and $\tau=40$. On figure 1 the input and output pump intensities are plotted when the rotation angle $\theta$ is $\pi \mathrm{t} / 100$. Such a choice leads clearly to $\rho^{+}(50)=0$ and the creation of a spike in the pump intensity profile. In effect, when $\rho^{+}$is cancelled, eq 19 shows that the pump intensity recovers since $\left|\tau^{+}\right|^{2}=1+\left|\rho^{+}\right|^{2}$ : this is the phenomenon of Raman spike formation. For large values of $\rho^{+}$, pump depletion occurs as shown again by eq. 19. In other words, this spike shows that the phenomenon of energy transfer between the pump and the Stokes is briefly inversed. This phenomenon has been experimentally observed [3-4].

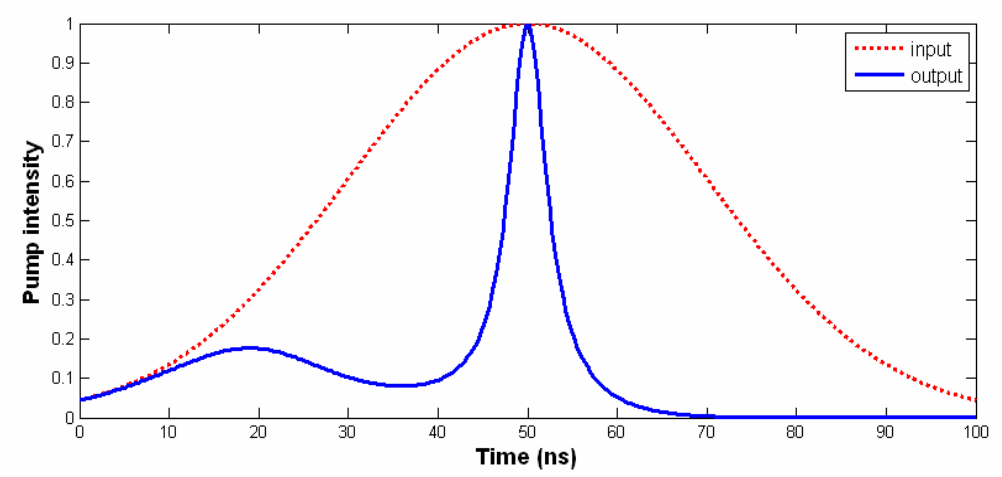

Figure $1:$ Input pump and output pump intensities. The parameters are $\tau=40, \Gamma=0.4, \theta=\pi \mathrm{t} / 100$

On figure 2, the multi-spike formation is shown to be possible when the polarization of the Stokes wave rotates several times in the duration of the pump input. Here, we choose $\theta=5 \pi \mathrm{t} / 100$. This phenomenon has not already been experimentally observed to the author's knowledge. 


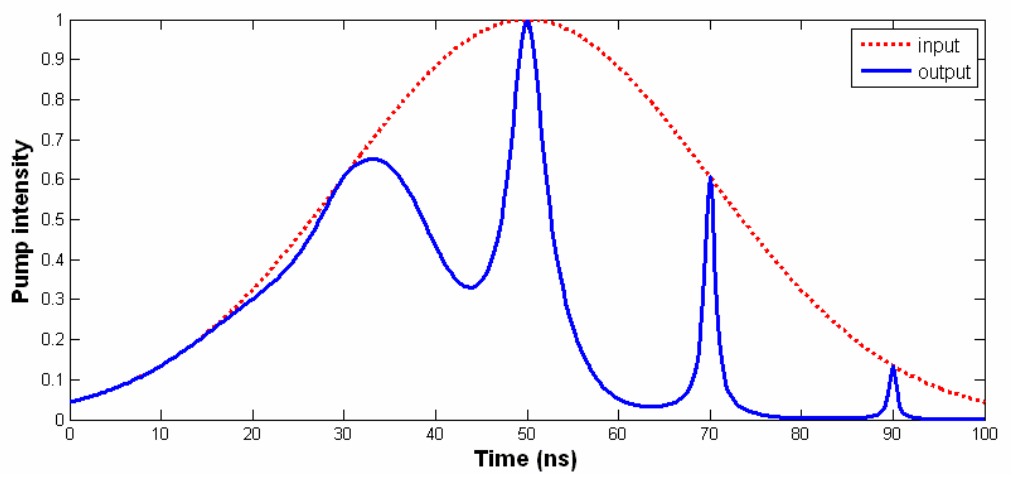

Figure 2 : Input pump and output pump intensities. The parameters are $\tau=40, \Gamma=0.4, \theta=5 \pi \mathrm{t} / 100$

\section{Conclusion}

In this work, we have considered the initial value problem of stimulated Raman scattering with vectorial optical fields. This problem has been shown to be solvable on the semi-line by the inverse scattering transform scheme. And in the limit x to infinity, explicit output waves envelopes have been obtained. Then the problem of soliton generation has been undertaken showing similar results to scalar SRS. However the general expression leading to solitons is much more complicated here when taking into account vectorial optical fields. Next, using the rotation of the Stokes wave polarization, the Raman spike and multi-spike formation is obtained in the time domain contrary to the scalar case. Its simple explanation is quite elegant. Finally, this work also shows that IST is a powerful tool to undertake a study on polarization. Such a study will be done in a future work.

\section{Acknowledgements:}

- The author would like to dedicate this work to the memory of Prof. J. Leon.

- This work was supported by CNRS contract 3073 GDR-PhoNoMi2 (Photonique Nonlinéaire et Milieux Microstructurés).

\section{References}

[1] A. Yariv, Quantum electronics, New-York Wiley 1975.

[2] R.W. Boyd, Nonlinear Optics, Academic Press, 2008.

[3] K. Drühl, R.G. Wenzel, and J.L. Carlsten, Phys. Rev. Lett. 51, 1171 (1983).

[4] K. Drühl, J.L. Carlsten and R.G. Wenzel, J. Stat. Phys., 39, 615 (1985).

[5] M.G. Raymer et J. Mostowski, Phys. Rev. A 24, 1980 (1981).

[6] I.A. Wamsley et M.G. Raymer Phys. Rev. Lett. 50, 962 (1983)

[7] N. Fabricius, K. Natterman et D. von der Linde, Phys. Rev. Lett. 52, 113 (1983).

[8] J.C. Englund et C.M. Bowden, Phys. Rev. Lett. 57, 2661 (1986)

[9] D.C. MacPherson, R.C. Swanson et J.L. Carlsten, Phys. Rev. A 40, 6745 (1989)

[10] J.C. Englund et C.M. Bowden, Phys. Rev. A 422870 (1990), 46, 578 (1992).

[11] M.J. Ablowitz et H. Segur, Solitons and the inverse scattering transform, SIAM, Philadelphia, 1981.

[12] S. Novikov, S.V. Manakov, L.P. Pitaevskii, V.E. Zakharov, Theory of solitons, the inverse scattering method, Math. Concepts and methods in science and engineering, Vol 7, 1984.

[13] F. Ginovart and J. Leon, Phys. Lett. A 194, 397 (1994) 
[14] C. Claude, F. Ginovart and J. Leon, Phys. Rev. A 52, 767 (1995)

[15] J. Leon and A.V. Mikhailov, Phys. Lett. A 253, 33 (1999).

[16] M. Boiti, J-G Caputo, J. Leon and F. Pempinelli, Inverse Problems 16, 303 (2000). 